\title{
Decline in direction in CASP experiments
}

\author{
Sergey Feranchuk
}

BACKGROUND. CASP experiment, "critical assessment of structure predictions", intended to discover advances in an ability of scientific groups to predict a structure of unknown protein from its sequence. The target sequences of proteins to be folded are chosen on each round. The challenge to fold a target from CASP is complicated and the structures of CASP targets are in some way different from an overall pool of known protein structures. The purpose of the study was to detect and quantify a difference between CASP targets and typical structures from the protein databank.

METHODS. An averaged local complexity of a protein fold was measured in units of entropy using several metrics which reduce a fragment of a fold to a binary distribution. A complexity was measured for targets from the previous rounds of CASP. A subset of PDB structures was prepared and an averaged complexity of PDB structures was estimated. The choice of the metrics in the measurement of complexity did simulate some of the approaches which were used to predict structures in CASP competition. A measurement of a modified complexity was performed, which was based on averaged distributions for fold fragments in common PDB structures.

RESULTS. A difference of CASP targets was detected by a metrics which uses hashing of distances between closely located residues. And a modified version of this metrics which emulates wide-range distance maps was shown to be most easily adjusted to utilize the difference between CASP targets and typical PDB structures. This means that, for the case of CASP targets, the methods which were trained on templates from PDB by similar metrices will guess the template structures in a new round of CASP more successfully with an increased gap in their ability to predict neutrally selected protein structures. This means that software, which relies on interresidue distances and performs well in CASP, will perform poorly in general-purpose structure prediction. 


\section{INTRODUCTION}

Targets and causes which drive a self-assembly of a protein for many years attract an attention of molecular biologists. The opinions there are sometimes reduced towards the idea that these drivers are hidden deeply in an "exact entropy-enthalpy-compensation pertaining to any structural changes induced in the solvent" [1].

CASP project, "critical assessment of structure predictions", was established in 1994. The project was positioned itself as an experiment which intended to detect abilities of modern scientific groups to predict 3D structure of proteins from the sequences.

13 rounds of the "experiment" have passed since 1994, the latest of comleteed rounds was in 2018. A several dozens of so-called "targets" were presented in each round; these targets are usually the sequences of proteins for which structure was already determined but a free access to it is postponed [2].

A problem of protein structure prediction is hard to solve and folds of proteins are complex. Several approaches were proposed to deal with that complexity. The usage of $6 x 6$ fragments of a contact map between adjacent protein residues was successful to compare protein structures [3]; hashing of triangles composed from atoms around active site allowed to guess similar sites in another proteins [4].

The reduction of a protein structure to its contact map was the most promising approach in early attempts of "template-free" structure prediction [5]. Following this way, it was recognized that to present a contact between residues as a binary value is not enough efficient and a more precise representation of a distance between closely located residues is required. This idea become to prevail, and, for example, in the approach [6] distances between residues were split to 64 bins. The method [6] allowed to provide the best template-free predictions in the latest round of CASP. The guess of a fold there was determined by neural networks which were traned on $64 \times 64$ fragments of a contact map.

The presented study provides a brief assessment of protein strictures which were used as targets at previous rounds of CASP. The comparison of complexity of the structures was performed, using several ways in which a protein fold can be reduced to a set of binary distributions. The trends observed for a complexity of different metrics were discussed, to point to possible illusions which decline the direction of CASP experiment from an assessment of advances in a discovery of protein folding problem. 


\section{METHODS}

Protein structures were analyzed which were presented as targets in 11 rounds of CASP, from 2 to 13 . Four types of metrics were used to assess a complexity of a structure. These were:

1. $6 \times 6$ square slices of a binary contact map;

2. distances in triangles of contacting residues, split to 2 bins;

3. distances from 2 to $10 \mathrm{~A}$, split to 32 bins;

4. distances from 2 to $10 \mathrm{~A}$, in the nodes of $6 \times 6$ square in contact map, split to 8 bins.

The processing of a whole protein chain applying any of the metrics provides a set of binary distributions and averaged values in each bin of a distribution were than converted to entropy measure. This value of entropy provided an estimate of a complexity of the structure. The properties of each metrics are provided below.

\begin{tabular}{|c|c|c|c|c|}
\hline Metrics & 1 & 2 & 3 & 4 \\
\hline number of bins & 36 & 7 & 32 & 32 \\
\hline threshold (in angstroms) & $<6$ & $>8$ & 2 to 10 & 2 to 10 \\
\hline Average enthropy: & \multirow{3}{*}{\multicolumn{4}{|c|}{$\begin{array}{llll}3.58349 & 1.44739 & 3.09717 & 3.12726 \\
3.58350 & 1.49703 & 3.14604 & 3.16783\end{array}$}} \\
\hline CASP targets & & & & \\
\hline subsample of PDB & & & & \\
\hline
\end{tabular}

The structures of CASP targets were used in the analysis, which were opened after the rounds were finished. In earlier rounds of CASP the identifiers of structures are available in the official site. For rounds from 11 to 13, "blastp" search in "pdbaa" blast database was applied to get the identifiers of structures with a complete identity with the target sequences. A total number of structures to be processed was 790 .

To estimate an average complexity of the structures in the protein databank (PDB), a set of 1000 non-redundant protein structures was selected from PDB. It was composed from representatives of protein clusters with 30\% identity available in RCSB site, 100 structures were taken for each two-year period from 2000 to 2020. 
A pieces of software on $\mathrm{C}++$ and python were prepared to run the analysis and to present the results, source codes of these utilities are available at doi://10.17605/OSF.IO/XMKSR "Inkscape" graphics editor was used to improve the appearance of the figures.

\section{RESULTS}

The distribution of enthropy measure for CASP targets is shown on fig. 1. Some decrease in the entropy of the distance-based metrices is observed in targets from CASP rounds 2 to 4. After several rounds of CASP, the idea of reconstruction of distance maps was approved to be most efficient; the performance of best approaches could influence the strategy by which targets for next round were chosen.

1

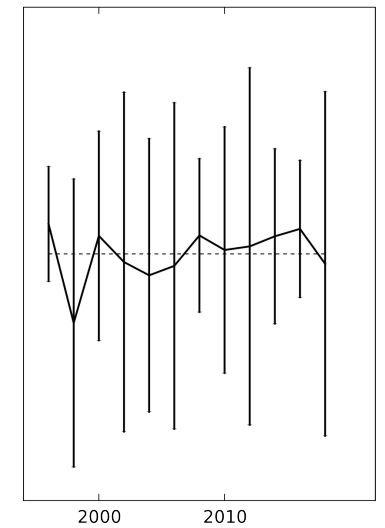

2

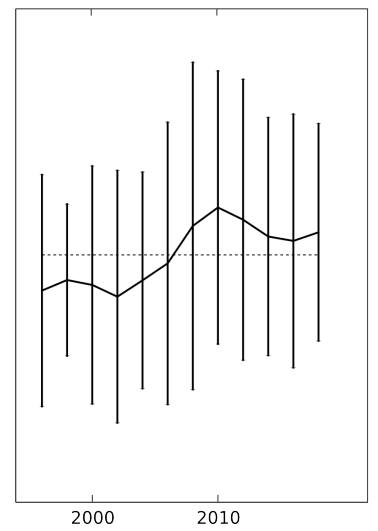

3

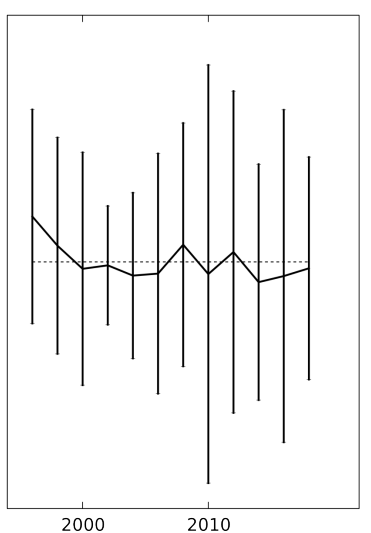

4

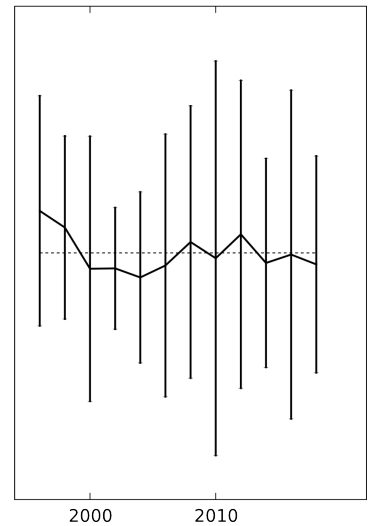

FIG. 1: Distribution of entropy measures for targets of 11 rounds of CASP. Error bars adjusted to standard deviation (SD) of a measure in each round. Margins in the charts adjusted to 2 SD from average across all targets. Labels on $\mathrm{X}$ axis are the years of the competitions.

The development of PDB itself is beyond the scope of this study. Anyway, any prediction software is trained on templates from PDB. A specific attention in a choice of templates for a training may be to the targets from previous rounds of CASP. This can decline the course in which a software for protein structure prediction is designed, which is developed by an intention to predict a structure from any protein sequence.

A value of entropy is a minimal quantity of information which encodes a complexity of a distribution. But a measure of complexity which can assess a difference of CASP targets from another structures is an information which is transmitted not in a code of the structure 
itself but in a common code of PDB templates. This quantity of information should be a bit greater than a conventional entropy. In the case of a provided brief analysis, it is expressed as $\sum p \log p_{0} ; p_{0}$ is a probability of a bin in a distribution, averaged through structures in PDB. So the distributions of entropy are shown on fig. 2 together with this updated quantity of information, in a scale adjusted to an average entropy of PDB structures.

1

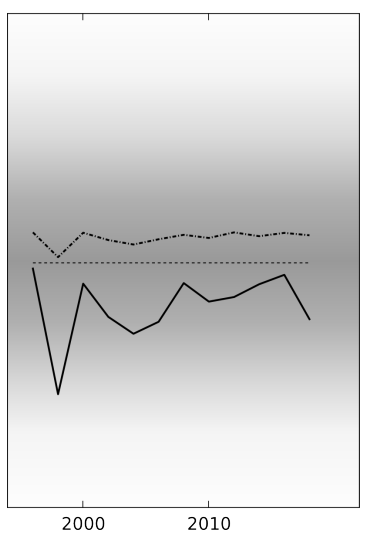

2

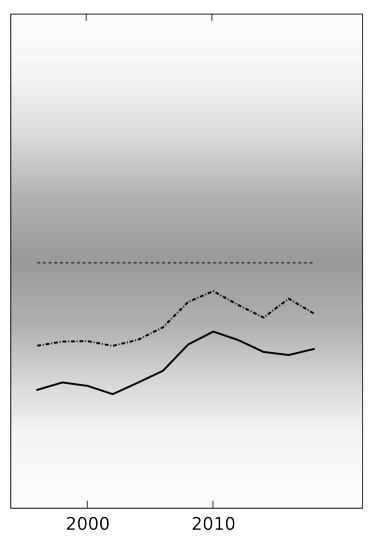

3

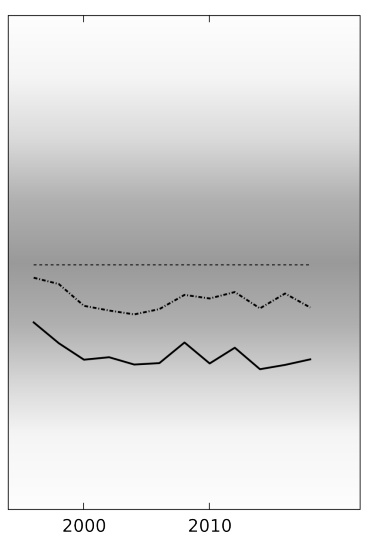

4

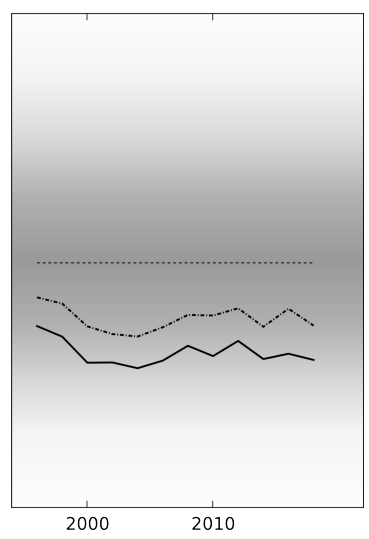

FIG. 2: Distribution of entropy measures (solid line) and of the relative quantity of information (dash-and-dot line), for the targets of CASP. Margins are adjusted to 2 SD from average in PDB and a shadow gradient in the background shows Gaussian distribution around that average.

The distance-based metrics for CASP targets are lower than for average in PDB, and this bias was not improved. And by the metric 4, from the two distance-based metrics, the relative entropy is the closest to true entropy. So, just for a case of CASP targets, the methods which were trained on templates from PDB by similar metrices will more successfully guess the template structures in a new round of CASP.

The motivation to choose targets in CASP is to avoid common folds which are widely presented in available experimental structures, and to select models which are in some way complicated to be folded by conventional routines. But this complexity may be a reduction relatively to an overall space of possible folds. A performance of previous CASP experiments should compensate the successes of over-simplified methods, but it can also provide a positive feedback. And indeed the approach to use $64 \times 64$ square slices which was used in [6] is better simulated by metric 4 . This mean that a much in a success in CASP competition is to find a feature that CASP targets have in common, to simplify a choice in the complex space of possible folds. And much of the efforts to develop well-performed software for CASP are 
indirect attempts to find the holes in the routine by which targets are selected.

\section{DISCUSSION}

Protein science is closer to physics and chemistry, than other major lineages of modern molecular biology. The solve how do proteins assemble, is a problem of physics and the solution could provide a solid bridge from molecular biology to these sciences. The true solution assumes an ability to explain how do protein fold into a globule, and to provide an effective model in a support of the explanation. This challenge motivated the establishment of CASP experiment.

But the trends observed in the CASP competitions mean like if it was assumed that a true solution of a template-free modeling do not exist. Better in this case is to try instead to find some "side-entry" in the approaches in which models for a competition are selected.

An entanglement in the Boltzmann-Gibbs meaning is accepted to be unresolvable. But were there provided any reasons why this unresolvable complexity is applicable to a folding of proteins? This is a case of disappointment, when not any sufficient evidence was declared to support it.

\section{ACKNOWLEDGMENTS}

The author is grateful to U. Potapova for the appreciation of his efforts.

[1] Ben-Naim A. Myths and verities in protein folding theories: from Frank and Evans icebergconjecture to explanation of the hydrophobic effect. J Chem Phys. 2013 Oct 28;139(16):165105

[2] Kryshtafovych A, Schwede T, Topf M, Fidelis K, Moult J. Critical assessment of methods of protein structure prediction (CASP)-Round XIII. Proteins. 2019 Dec;87(12):1011-1020

[3] Holm L, Sander C. Protein structure comparison by alignment of distance matrices. J Mol Biol. 1993 Sep 5;233(1):123-38

[4] Shulman-Peleg A, Nussinov R, Wolfson HJ. Recognition of functional sites in protein structures. J Mol Biol. 2004 Jun 4;339(3):607-33. 
[5] Contact order and ab initio protein structure prediction. Bonneau R, Ruczinski I, Tsai J, Baker D. Protein Sci. 2002 Aug;11(8):1937-44.

[6] Senior AW, Evans R, Jumper J, Kirkpatrick J, Sifre L, Green T, Qin C, dek A, Nelson AWR, Bridgland A, Penedones H, Petersen S, Simonyan K, Crossan S, Kohli P, Jones DT, Silver D, Kavukcuoglu K, Hassabis D. Protein structure prediction using multiple deep neural networks in the 13th Critical Assessment of Protein Structure Prediction (CASP13). Proteins. 2019 Dec;87(12):1141-1148 